\title{
Study and Optimization of Self-Assembled Polymeric Multilayer Structures with Neutral Red for pH Sensing Applications
}

\author{
Javier Goicoechea, Francisco J. Arregui, Jesus M. Corres, and Ignacio R. Matias \\ Departamento de Ingeniería Eléctrica y Electrónica, Universidad Publica de Navarra, Campus Arrosadia, 31006 Pamplona, Spain
}

Correspondence should be addressed to Javier Goicoechea, javier.goico@unavarra.es

Received 21 August 2007; Accepted 1 October 2007

Recommended by Andrea Cusano

\begin{abstract}
The characterization of nanostructured thin films is critical in the design and fabrication of optical sensors. Particularly, this work is a detailed study of the properties of layer-by-layer electrostatic self-assembled multilayer (LbL) structures fabricated using poly(allylamine hydrochloride) (PAH) and Neutral Red (NR) as cations, and poly(acrylic acid) (PAA) as polyanion. These LbL films, due to the colorimetric properties of the NR, are suitable for sensor applications such as $\mathrm{pH}$ sensing in the physiological range. In the (PAH+NR/PAA) LbL structure, it has been observed a very important influence of the $\mathrm{pH}$ of the solutions in the properties of the resultant films. Different techniques such as spectroscopy and atomic force microscopy (AFM) are combined to characterize the films, and the results are analyzed showing coherence with previous works. The LbL structure is finally optimized and dramatically improved nanostructured films were fabricated, showing good sensing properties, short response times, and good stability.
\end{abstract}

Copyright (C) 2008 Javier Goicoechea et al. This is an open access article distributed under the Creative Commons Attribution License, which permits unrestricted use, distribution, and reproduction in any medium, provided the original work is properly cited.

\section{INTRODUCTION}

Optical sensing is becoming a real alternative to the conventional sensors in some applications because of their important advantages as electromagnetic immunity, biocompatibility, multiplexing, miniaturizing capability, and so forth. Nanostructured thin-films have demonstrated to be one of the most promising approaches for optical sensor solutions as far as it is possible to tailor their properties and sensitivity, and they can be fabricated onto a lot of optical instruments, for example, lenses, prisms, even optical fibers. One powerful feature of nanostructured materials is the tunability of the molecular-scale composition, leading to new composite materials with very interesting properties resulting from a smart design. In fact, the number of scientific publications and patents in this topic has risen exponentially since 1985 [1]. Several techniques are commonly used in the fabrication of these nanostructured thin films, like CVD [2], thermal vacuum deposition [3], spin-coating [4], Langmuir-Blodgett (LB) technique [5], layer-by-layer electrostatic self-assembly method (LbL), and so forth. More specifically, the LbL tech- nique, first reported by Decher [6], is a very versatile tool useful in creating multilayer structures in the nanometric range. With this technique it is possible to tune the composition of the layers at molecular scale, fabricate interesting composite materials in a simple and inexpensive way onto substrates composed by almost any material and with almost any size or shape. This technique has been successfully demonstrated in many applications such as conductive coatings [7], antireflective coatings [8], sensitive coatings [9-14], organic light emitting diodes [15], photovoltaic cells [16], and so forth.

In the past, several approaches have been reported for $\mathrm{pH}$ optical sensing like the use of lenses or optical components coated with $\mathrm{pH}$ indicators [17], sol-gel matrices with fluorescent dyes [18], absorbance $\mathrm{pH}$ indicators [19], or polystyrene microspheres [20,21], just to give some examples. This study is focused on the characterization of polymeric LbL multilayer coatings for optical fiber sensors. These LbL sensors offer some advantages like high quality of the optical films, reproducibility, miniaturization of the probes, fast response, biocompatibility, and dynamic range within the physiological range. These properties make them suitable for in vivo 
applications, for example. This work is an optimization of the properties of LbL films fabricated using poly(allylamine hydrochloride) (PAH) as polycation and poly(acrylic acid) (PAA) as polyanion, adding to this polymeric matrix neutral red (NR), a cationic $\mathrm{pH}$ indicator. These LbL films follow the structure PAH + NR/PAA. Previous works of Rubner and his group on the PAH/PAA LbL multilayer films were used as reference [22-25]. The addition of a third material, NR, during the self-assembly process modifies the properties of the LbL films, so the resultant films are suitable for sensing applications in the physiological range, as it was experimentally demonstrated in [13]. Moreover, some fabrication parameters have a dramatic influence in the thickness and morphology of the LbL films, for example, the immersion time [26], the temperature [27], the polyelectrolyte concentration, and ionic strength of the solutions [28], the $\mathrm{pH}$ of the solutions when working with weak polyelectrolytes [23], and so forth. The aim of the present work is to characterize the resultant $\mathrm{PAH}+\mathrm{NR} / \mathrm{PAA}$ LbL films, and to find optimal experimental conditions for achieving the higher concentration of the $\mathrm{pH}$ indicator, NR, in the sensitive films.

The experimental study involves several steps that will be communicated below. In a first approach of the analysis, it is important to know the pKa value of the materials involved in the LbL process in order to understand how the molecules behave during the assembly process. Afterwards, some LbL films were fabricated at different $\mathrm{pH}$ values and their thickness and composition were measured in order to characterize the PAH + NR/PAA structures. The discussion is structured into two main parts: the first one is an AFM study of the films as the $\mathrm{pH}$ of the solutions is varied, which gives information about the thickness and roughness of the films, and the second part is a spectroscopy study of the same films which give information of their composition. Finally, the analysis of the experimental results will lead us to an optimization of the NR concentration of the LbL films. To our knowledge this is the first experimental study performed to optimize this kind of polymeric structure towards sensing applications.

\section{EXPERIMENTAL}

\subsection{Materials}

The polyelectrolytes in this work are poly(allylamine hydrochloride) (PAH) as the polycation and poly(acrylic acid) (PAA) as the polyanion. The active dye used in this work is neutral red (NR). All the chemicals were purchased from Sigma-Aldrich Quimica (S.A.) and were used without any further purification. Since NR is a cationic small molecule, it will be added to the polycationic solution $(\mathrm{PAH}+\mathrm{NR})$ rather than to the PAA to avoid aggregation with the polyanion, which could led to inhomogeneous layers.

\subsection{LbL process}

Dilute aqueous solutions of PAH, NR, and PAA $\left(10^{-2} \mathrm{M}\right.$ based on the repetitive unit molecular weight) were prepared. Afterwards, the PAH and NR solutions were mixed so the molar ratio of NR is fixed to a $6.5 \%$ respect to the PAH.
The $\mathrm{pH}$ value of the solutions was adjusted to the desired value by adding a few drops of $\mathrm{HCl}$ or $\mathrm{NaOH}$. The $\mathrm{pH}$ of the polyelectrolyte solutions was varied throughout all the experiments, sweeping from 2.5 to 9.5 , keeping the $\mathrm{pH}$ of both polycationic and polyanionic solutions equal in each experiment. For $\mathrm{pH}$ values lower than 2.5 and higher than 9.5 the polymers are not stable and the final films showed serious flaws and inhomogeneities. The substrates used in this work are standard glass microscope slides with a pretreatment of immersion in a solution of $\mathrm{H}_{2} \mathrm{SO}_{4}: \mathrm{H}_{2} \mathrm{O}_{2}$ (7:3 vol.) and afterwards thoroughly rinsed in ultrapure water. Then the substrates were immersed for 2 minutes in the polyelectrolyte solution, they were rinsed in two separate beakers of ultrapure water for 1 minute each, and afterwards the same protocol was performed using the counter-polyelectrolyte solution, completing a bilayer (a polycation/polyanion layer block). The final structure can be written as $(\mathrm{PAH}+\mathrm{NR} / \mathrm{PAA})_{n}$, where $n$ is the number of bilayers. For this experiment, 25 bilayers were chosen unless otherwise stated. The fabrication of the multilayer films was carried out using a 3-axis cartesian dipping robot $(\mathrm{R} \& \mathrm{~K} \mathrm{GmbH})$ with the dipping protocol detailed before.

\subsection{Thickness and roughness measurements}

An AFM digital instruments NanoScope IIIa Multimode in tapping mode was used. The thickness measurements were performed making a scratch in the films with a razor blade, and then measuring the depth of the line. The roughness of the films was also measured with the AFM in tapping mode, with silicon cantilever (RTESP, Veeco) in a region near the central zone of the sample to avoid border effects.

\subsection{Absorbance measurements}

The absorbance spectra of the films were measured using a typical transmission fiber optic setup with an OceanOptics DH-2000-S white light source (from $200 \mathrm{~nm}$ to $1500 \mathrm{~nm}$ ), and an OceanOptics USB4000 Spectrometer.

\section{RESULTS AND DISCUSSION}

As it has been previously introduced, the degree of ionization of the polyelectrolytes depends on the $\mathrm{pH}$ of the surrounding medium. This degree of ionization affects the proportion of free ionic binding sites, and consequently to the morphology of the polymer chains. Therefore, the thickness and roughness of the LbL films are strongly dependent on the $\mathrm{pH}$ of the polyelectrolyte solutions [20-25]. In the present work, the self-assembly of PAH and PAA processes is altered by the addition of the neutral red (NR) to the polycationic solution. The thickness results of the $(\mathrm{PAH}+\mathrm{NR} / \mathrm{PAA})_{n}$ structure were studied to characterize the films, and the previous work with $(\mathrm{PAH} / \mathrm{PAA})_{n}$ reported by Shiratori and Rubner [23] was taken as reference in the following analysis.

In the PAH/PAA system, it has been demonstrated [2025 ] that the thickness of the bilayers is affected not only by the conformation of the adsorbing polyelectrolyte, but also by the density of electrostatic charge and the thickness of the 


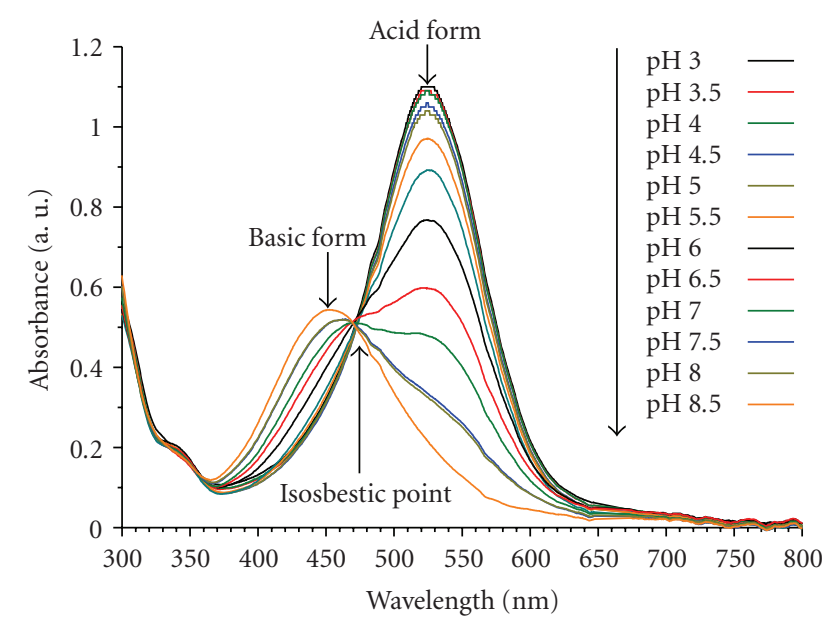

FIgURE 1: Absorption spectra of NR solutions at different $\mathrm{pH}$ values.

previous layer. Therefore, there is a complex interaction between the two-weak polyelectrolytes depending on the $\mathrm{pH}$ value of the solutions. In the analysis of the PAH/PAA system, the pKa of the polyelectrolytes is a key factor because it gives information about the degree of ionization of the electrolyte. As reported in [25] the pKa of PAH is approximately 8.8 , and the PAA has a $\mathrm{pKa}$ of 6.5 . That means that PAH will be fully ionized at low $\mathrm{pH}$ values (lower than $\mathrm{pH}$ ) and completely de-ionized at $\mathrm{pH} 12$. In the case of the polyanion, a reverse behavior is observed; the PAA is fully ionized at high $\mathrm{pH}$ values ( $\mathrm{pH} 9.5$ or $\mathrm{pH} 10$ ), and almost completely deionized at $\mathrm{pH}$. As the $\mathrm{pH}$ value of polyelectrolyte solutions is varied, different incremental thicknesses per bilayer are achieved. The (PAH/PAA) bilayer thickness versus $\mathrm{pH}$ plot shows a characteristic shape [23], with a relative maximum around $\mathrm{pH} 4.5$, followed by a dramatic decrease in thickness between pH6 and pH7.5, and then another drastic increase for $\mathrm{pH}$ values higher than $\mathrm{pH} 8$. The thicker films occur when one of the polyelectrolytes is not completely ionized while the counter-polyelectrolyte is in its fully ionized form. On the other hand, when both polyelectrolytes are almost fully ionized, the thinnest films were achieved.

In this work, the PAH is mixed with NR, so during the immersion of the substrate into the positive solution, both PAH and NR will compete for the free binding sites in the PAA. It is very important to know the pKa value of the NR in order to analyze the role that NR plays in the new $\mathrm{PAH}+\mathrm{NR} / \mathrm{PAA}$ system. In order to calculate the pKa of NR, a similar procedure as in [25] is adopted and the absorption spectrum of NR diluted in water at different $\mathrm{pH}$ values is measured, see Figure 1.

In this plot, there is three remarkable points, around $450 \mathrm{~nm}$ and $525 \mathrm{~nm}$ where the basic and acid species absorption peaks are around $470 \mathrm{~nm}$ where the isosbestic point is located. From these spectra, the deprotonated and protonated species of NR were taken as references for the absorbance measurements, thus the absorbance at $450 \mathrm{~nm}$ and $525 \mathrm{~nm}$ was taken for calculating the degree of ionization as is described below. We considered that at very low $\mathrm{pH}$ val-

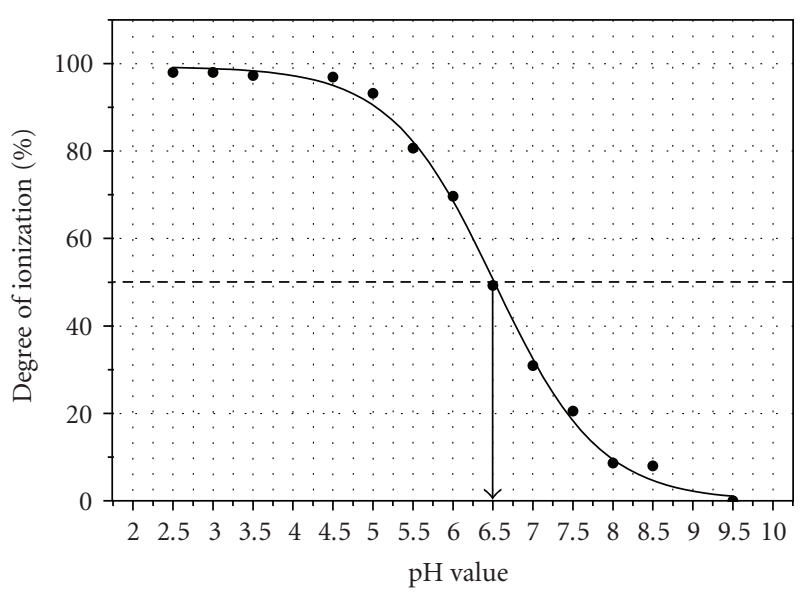

FIGURE 2: Ionization degree of the NR in aqueous solution versus the $\mathrm{pH}$.

ues ( $\mathrm{pH} 2.5$ ), the NR molecules were fully charged, whereas at very high $\mathrm{pH}$ values ( $\mathrm{pH} 9.5$ ) the NR is completely deprotonated. Then, the ratio $\left(A_{525 \mathrm{~nm}} / A_{450 \mathrm{~nm}}\right)$ at $\mathrm{pH} 2.5$ is taken as the $100 \%$ ionization rate, and $\left(A_{525 \mathrm{~nm}} / A_{450 \mathrm{~nm}}\right)$ at $\mathrm{pH} 9.5$ is taken as the $0 \%$. Therefore the degree of ionization (DI) is defined according to [25]

$$
\mathrm{DI}=\frac{\left(A_{525 \mathrm{~nm}} / A_{450 \mathrm{~nm}}\right)_{\mathrm{pH}}-\left(A_{525 \mathrm{~nm}} / A_{450 \mathrm{~nm}}\right)_{\mathrm{pH}=9.5}}{\left(A_{525 \mathrm{~nm}} / A_{450 \mathrm{~nm}}\right)_{\mathrm{pH}=2.5}-\left(A_{525 \mathrm{~nm}} / A_{450 \mathrm{~nm}}\right)_{\mathrm{pH}=9.5}} \times 100 \% \text {. }
$$

Figure 2 displays the degree of ionization versus the $\mathrm{pH}$ of the NR solution, and the pKa can be calculated as the $\mathrm{pH}$ in which the NR is half ionized, giving an approximate value of $\mathrm{pKa}=6.5$. This value of $\mathrm{pKa}$ is consistent with other values found in the bibliography [29]. According to the weak electrolyte nature of the NR and its pKa value, it is predictable that it would be more likely to be adsorbed in a LbL process at $\mathrm{pH}$ values under 6.5 , because in more acidic solutions it would be in its protonated state, and consequently it would show affinity for the free negative binding sites available at the PAA. This hypothesis is confirmed by the experimental data, as will be shown later.

After this previous study about the $\mathrm{pKa}$ of $\mathrm{NR}$, the $(\mathrm{PAH}+\mathrm{NR} / \mathrm{PAA})_{n}$ coatings were built on microscope glass slides and the $\mathrm{pH}$ of the cationic and anionic solutions used for the fabrication of the films was varied from 2.5 to 9.5 in steps of $0.5 \mathrm{pH}$ units. Then, in order to calculate the amount of NR molecules added to the LbL films, the resultant thickness of the $(\mathrm{PAH}+\mathrm{NR} / \mathrm{PAA})_{n}$ films were studied by AFM and spectroscopy.

In Figure 3, AFM pictures of two different LbL assemblies fabricated at pH2.5 and 3.5 are displayed as an example of the results obtained. From these results it can be deducted that the roughness of the resultant layers can also be optimized by simply varying the $\mathrm{pH}$ of the solution, which is critical in certain applications as in long period grating (LPG) based on nanostructured coatings [30]. The experimental results of the AFM analysis are shown in Figure 4, where it is 


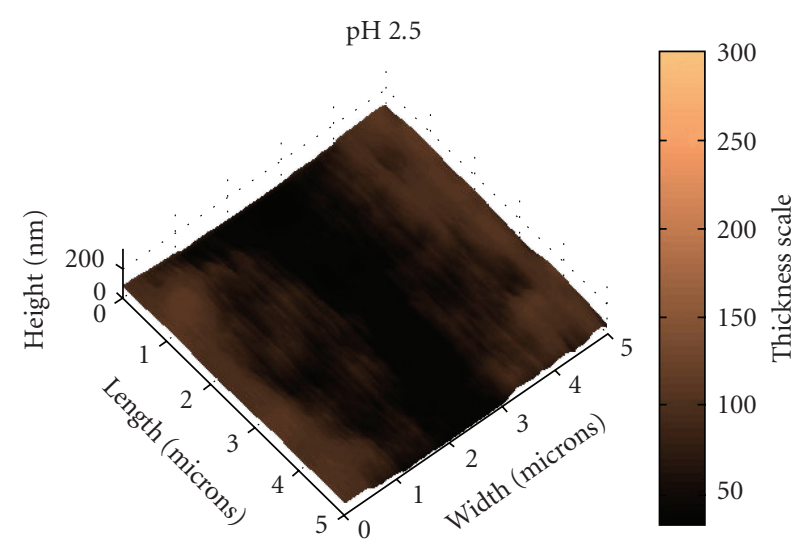

(a)

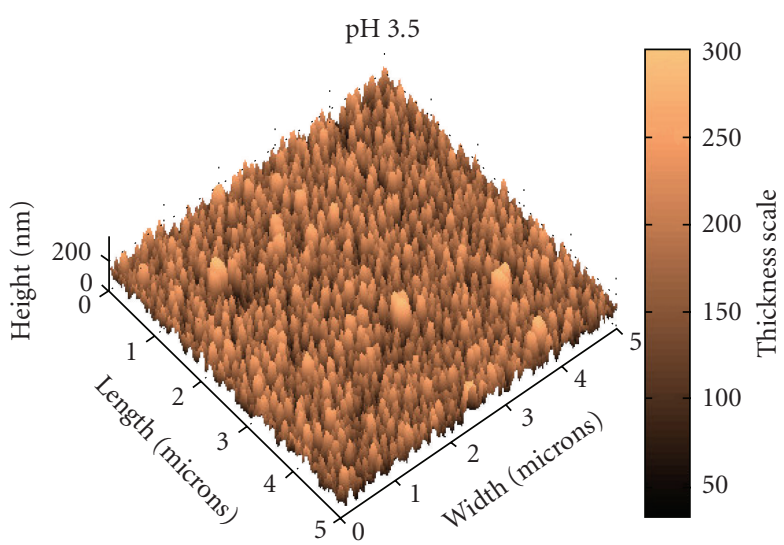

(b)

FIGURE 3: AFM pictures of two (PAH + NR/PAA) 25 structures built up at different $\mathrm{pH}$ values.

displayed the evolution of thickness and roughness as the $\mathrm{pH}$ of the fabrication $\mathrm{pH}$ is varied. This useful information allows us to determine the optimal conditions of thickness or roughness for the final sensitive films. Nevertheless, in this particular application, the focus is placed in the thickness of the multilayer coatings rather than in their roughness as far as the concentration of NR respect to the polymeric matrix is being optimized. The thickness versus $\mathrm{pH}$ profile shown in Figure 4 is very similar to the (PAH/PAA $)_{n}$ structure reported by Shiratori and Rubner in [23] due to the weak polyelectrolyte nature of the materials involved in the LbL process. However, some important differences are found here with respect to that structure. In the $(\mathrm{PAH}+\mathrm{NR} / \mathrm{PAA})$ structures there is a relative thickness maximum around $\mathrm{pH} 5.5$ which is slightly right-shifted compared to the (PAH/PAA) structure [23]. In addition, a thickness minimum region between pH6.5 and pH7.0, but layers, are thicker than that in the (PAH/PAA) case. This is logical since the presence of NR implies a higher ionic strength of the polyionic solutions and, therefore a higher thickness of the resulting coatings [28].

The spectroscopy study performed on the films is shown in Figures 5 and 6. Figure 5 displays the absorbance spectra of different LbL films as the $\mathrm{pH}$ value of the polyelectrolyte solutions is varied. This plot shows that the absorption spec-

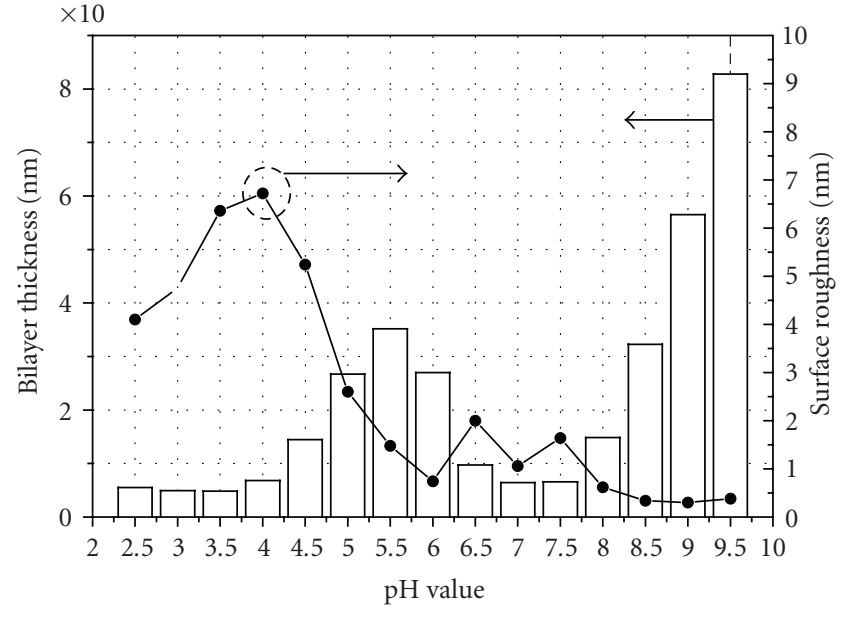

FIGURE 4: Left: the bars show the bilayer thickness as the $\mathrm{pH}$ of the polyelectrolyte solutions was varied. Right: the dotted line is the square roughness of the surface of the $(\mathrm{PAH}+\mathrm{NR} / \mathrm{PAA}) 25$.

tra of the films from pH6 to pH9.5 are almost flat, so the films are very transparent, revealing no presence of NR in the films. From pH2.5 to pH5.5 the spectra show a clear peak around $520 \mathrm{~nm}$, which corresponds to the protonated NR absorption peak. A detailed observation of these spectra shows a slight displacement of the NR peak to lower wavelengths as the $\mathrm{pH}$ value is increased from $\mathrm{pH} 2.5$ to $\mathrm{pH} 5.5$. This left shift of the peak is due to the color change of the NR indicator itself as consequence of the increasing of the $\mathrm{pH}$. As far as the objective of this spectroscopic study is to calculate the amount of NR contained in the LbL films, the absorbance at the isosbestic point of the NR spectrum (around $470 \mathrm{~nm}$ ) is taken as indicator in order to avoid any interference of the peak shift. In Figure 6 it is shown the evolution of the absorbance at $470 \mathrm{~nm}$ as the $\mathrm{pH}$ of the solutions is varied which gives an idea of the amount of NR in the polymeric films. This absorption at the isosbestic point of NR showed in Figure 6 suggests that NR molecules are being incorporated to the LBL structure only at low $\mathrm{pH}$ values of the solutions, which hold well with the hypothesis previously formulated keeping in mind that NR has a pKa around 6.5.

The absorption at the isosbestic point of the NR gives information about the total amount of NR contained in the films, regardless of their thickness. It has to be noticed that the film which presents a highest absorbance at $470 \mathrm{~nm}$ is not the thickest one. Comparing the data shown in Figures 4 and 6 , it can be seen that the maximum thickness in Figure 4 happens at pH5.5, while the maximum absorbance in Figure 6 is at pH4.5. This is because a thicker bilayer does not imply necessarily a higher adsorption of NR and it is due to the difference between the pKa values of the PAH and the NR. Taking as starting point $\mathrm{pH} 2.5$ and increasing the $\mathrm{pH}$, the number of binding sites in the PAA is increased, leading to thicker layers. In this $\mathrm{pH}$ range the $\mathrm{PAH}$ and the NR compete for the charged sites in the PAA. The NR molecules probably due to their size seem to be more likely to be adsorbed onto the PAA leading to slightly thinner layers with high concentration of the dye. When the $\mathrm{pH}$ reaches values higher than 4.5 , 


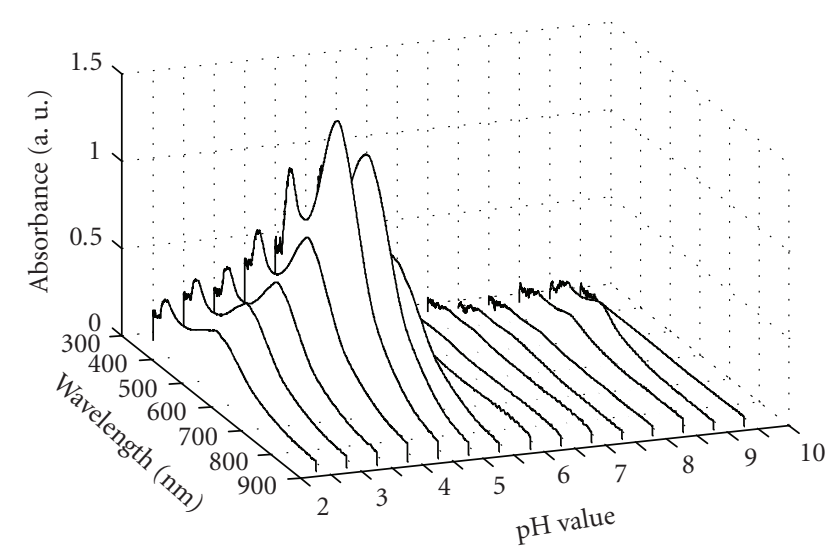

FIGURE 5: Absorption spectra of (PAH + NR/PAA)25 fabricated with polyelectrolyte solutions at different $\mathrm{pH}$ values.

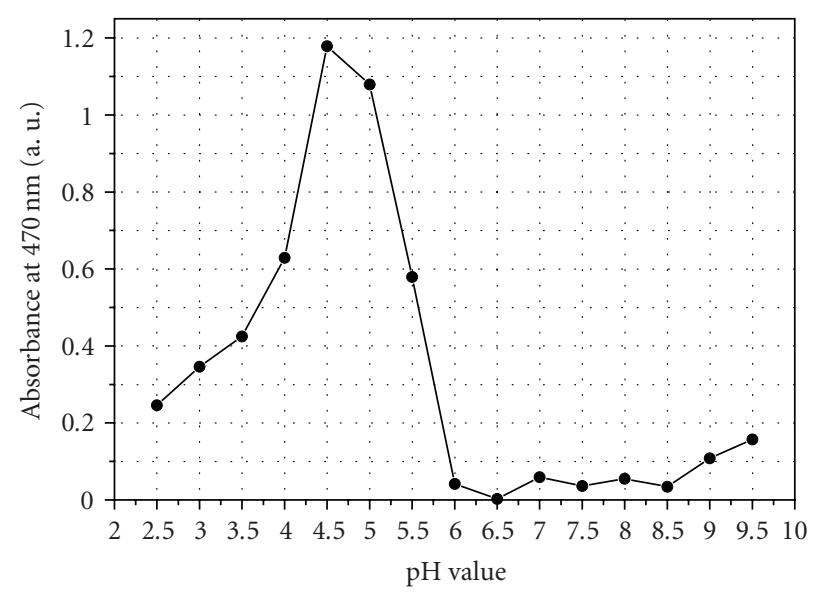

Figure 6: Comparison between the average bilayers thickness and the absorbance at $470 \mathrm{~nm}$. This comparison shows evidence that the composition of the $(\mathrm{PAH}+\mathrm{NR} / \mathrm{PAA}) 25$ changes as $\mathrm{pH}$ is increased.

the degree of ionization of NR molecules drops abruptly (see Figure 2). Consequently, the concentration of NR in respect to the PAH decreases while PAH is still almost fully ionized and it still forms thick layers. The result is a variation in the NR richness of the resultant film. In order to have a measurement of the NR adsorbed per unit of volume it is necessary to calculate the ratio between absorption at $470 \mathrm{~nm}$ and the thickness of the film. This ratio is equivalent to the linear absorption coefficient $(\alpha)$ at $470 \mathrm{~nm}$, defined as

$$
\alpha_{470 \mathrm{~nm}}=\frac{\text { Absorbance }_{470 \mathrm{~nm}}}{\text { TotalFilmThickness }_{\text {avrg }}} .
$$

Figure 7 displays the evolution of the $\alpha_{470 \mathrm{~nm}}$ of the films as the $\mathrm{pH}$ of the polyelectrolyte solutions is varied. The maximum of this ratio is the optimal film composition, as far as it contains the highest concentration of NR per unit of volume, and this happens at pH4.0. The experimental data collected from the films built-up at different $\mathrm{pH}$ value are summarized in Table 1. This complete characterization of the films, taking into account not only the total absorbance but also the thickness, is very important in the design of many optical devices

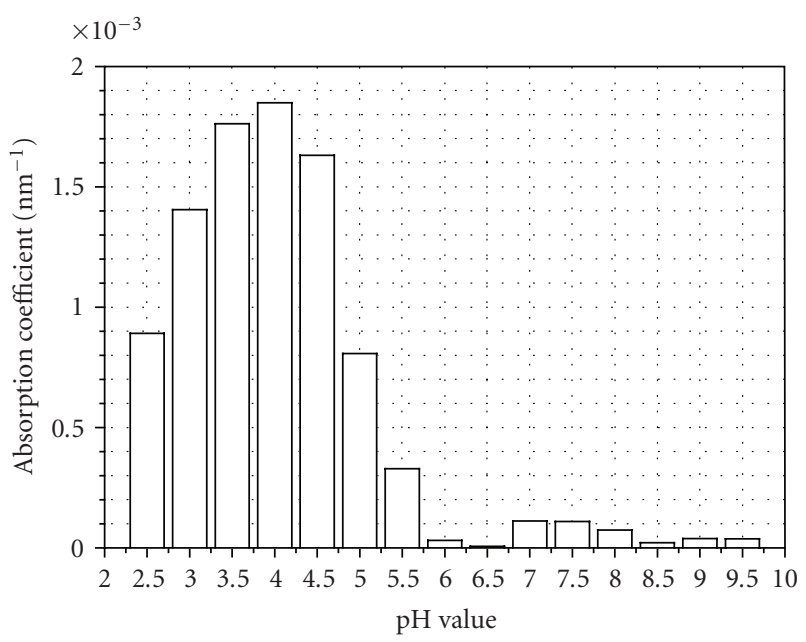

Figure 7: Absorption coefficient at $470 \mathrm{~nm}$ versus $\mathrm{pH}$ of the polyelectrolyte solutions. A maximum concentration of NR is found for the films built-up at pH4.0.

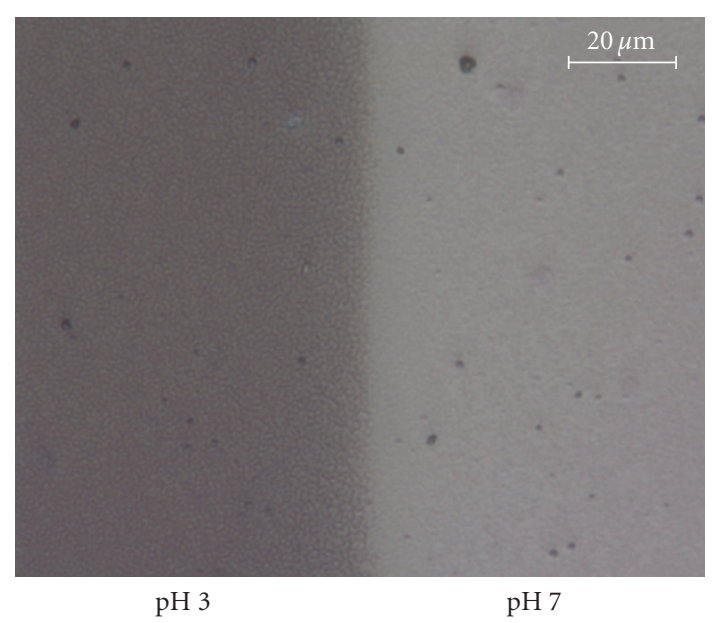

Figure 8: Micrograph of a (PAH + NR/PAA) 25 coating fabricated at $\mathrm{pH} 4$ partially immersed into $\mathrm{pH} 3$ (left) and $\mathrm{pH}$ (right) buffer solutions. Change of color of the coating is fast (few seconds) and reversible.

like filters or optical fiber sensors where both the thickness of the layers and the concentration of the active molecules (NR in this case) are necessary in order to achieve a proper design [13].

In order to corroborate that the NR indicator still kept its sensing properties once embedded in the polymeric matrix, the $(\mathrm{PAH}+\mathrm{NR} / \mathrm{PAA})$ optimized coatings were immersed into different $\mathrm{pH}$ buffer solutions and a dramatic change of color was observed, as is displayed in Figure 8. In fact, the coatings showed an optical response between the acid state (red) and the basic state (yellowish) with fast response times, in the order of just a few seconds.

Finally, some fiber optic sensor was fabricated and tested. The aim of this test is only to prove the good sensing properties of the optimized LbL films in a real application, but 
TABLE 1: Measurements of the thickness, roughness, and absorbance at $470 \mathrm{~nm}$. The absorption coefficient at $470 \mathrm{~nm}$ was calculated from these data.

\begin{tabular}{|c|c|c|c|c|}
\hline $\mathrm{pH}$ of the solutions & Bilayer thickness (nm) & Surface roughness $(\mathrm{nm}, \mathrm{rms})$ & Absorbance at $470 \mathrm{~nm}$ (a.u.) & Absorption coefficient $\left(\mathrm{nm}^{-1}\right)$ \\
\hline 2.5 & 5.5 & 4.12 & 0.246 & 0.00089 \\
\hline 3.0 & 4.9 & 4.76 & 0.346 & 0.00141 \\
\hline 3.5 & 4.8 & 6.36 & 0.425 & 0.00176 \\
\hline 4.0 & 6.8 & 6.72 & 0.629 & 0.00185 \\
\hline 4.5 & 14.5 & 5.24 & 1,179 & 0.00163 \\
\hline 5.0 & 26.7 & 2.61 & 1,079 & 0.00081 \\
\hline 5.5 & 35.2 & 1.48 & 0.579 & 0.00033 \\
\hline 6.0 & 27.0 & 0.74 & 0.042 & 0.00003 \\
\hline 6.5 & 9.7 & 2.04 & 0.003 & 0.00001 \\
\hline 7.0 & 6.4 & 1.06 & 0.059 & 0.00011 \\
\hline 7.5 & 6.6 & 1.64 & 0.036 & 0.00011 \\
\hline 8.0 & 14.8 & 0.62 & 0.055 & 0.00007 \\
\hline 8.5 & 32.3 & 0.34 & 0.034 & 0.00002 \\
\hline 9.0 & 56.6 & 0.31 & 0.108 & 0.00004 \\
\hline 9.5 & 82.8 & 0.38 & 0.157 & 0.00004 \\
\hline
\end{tabular}

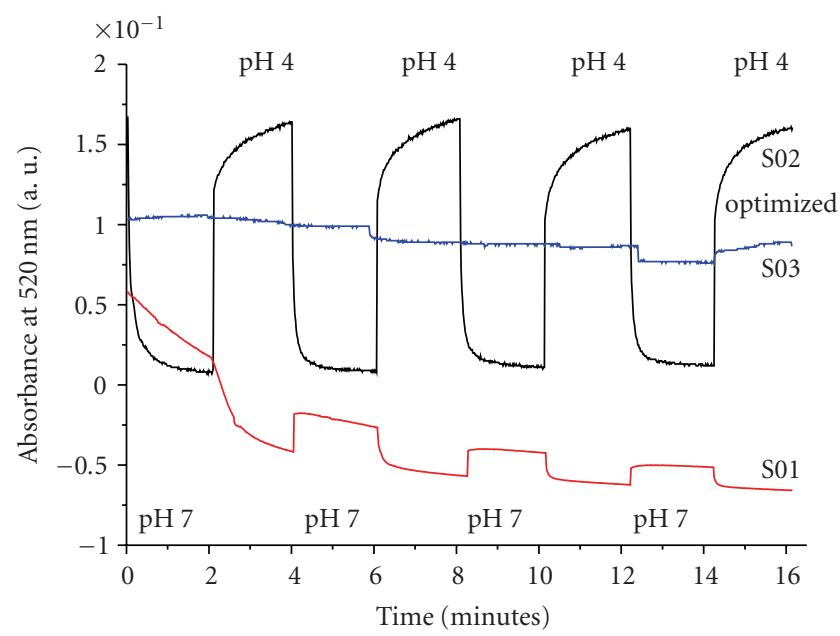

FIGURE 9: Dynamic response of three sensors consisting in a $(\mathrm{PAH}+\mathrm{NR} / \mathrm{PAA}) 25$ fabricated at three different $\mathrm{pH}$ values. S01 was fabricated at $\mathrm{pH} 7, \mathrm{~S} 02$ at $\mathrm{pH} 3$, and $\mathrm{S} 03$ at $\mathrm{pH} 4$. $\mathrm{S} 03$ response was found to be the most sensitive and stable signal.

the sensor setup is not optimized and its performance can be enhanced in future works. The sensor multilayer structures were built-up onto the end-face of cleaved standard telecommunication multimode optical fibers (62.5 and 125 microns of core and cladding diameter, resp.) following the procedure described in the experimental section, and using a $\mathrm{pH}$ value of the polyelectrolyte solutions of 3, 4, and 7 (noted as S01, S02, and S03, resp.). Afterwards the tip of the different fibers was immersed into $\mathrm{pH} 4$ and $\mathrm{pH} 7$ buffer solutions and the reflected optical power was registered using a CCD spectrometer. In Figure 9, the dynamic response of the three different sensors is displayed. This figure clearly shows how the sensibility and stability of the sensitive LbL coating can be optimized when the nanostructure properties are properly ad-

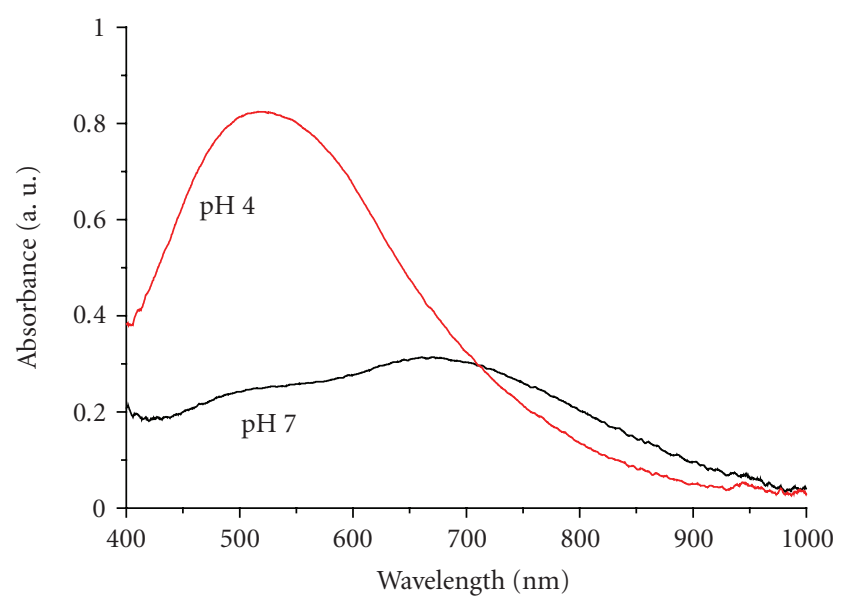

FIGURE 10: Spectral response of an optical fiber/(PAH + NR/PAA $)_{25}$ sensor fabricated at $\mathrm{pH} 4$.

justed. Figure 10 shows the optical response from the optical fiber sensor fabricated at $\mathrm{pH} 4$. As it can be seen, the spectral response of the sensor shows a broad absorption band around $520 \mathrm{~nm}$ when it is immersed in $\mathrm{pH}$, that vanishes when the $\mathrm{pH}$ is changed to $\mathrm{pH} 7$, corresponding to the typical behavior of NR. The response of the sensor was found to be fast and very repetitive, suggesting good stability of the LbL sensing films.

\section{CONCLUSIONS}

PAH + NR/PAA LbL coatings was studied and optimized for $\mathrm{pH}$ sensing. As far as all the materials are weak electrolytes, the $\mathrm{pH}$ of the aqueous solutions used for building up the films plays a key role in the final properties of the assemblies. Some conclusions about the interaction of the molecules during the LbL process have been extracted by combining 
spectroscopic measurements with AFM morphological analysis of the films. It was found that the concentration of NR molecules adsorbed into the LBL films and the bilayer thickness as well strongly depends on the $\mathrm{pH}$ of the polyelectrolyte solutions used for the fabrication of the coatings. An optimization of the absorption linear coefficient was performed achieving a maximum when the $\mathrm{pH}$ of the polyelectrolyte solutions is fixed to 4.0. Therefore, in order to obtain sensitive $\mathrm{PAH}+\mathrm{NR} / \mathrm{PAA}$ LbL coatings with a maximum adsorption of $\mathrm{NR}$, the nanostructured films have to be fabricated at $\mathrm{pH} 4$.

\section{ACKNOWLEDGMENTS}

This work was supported in part by the Spanish CICYTFEDER Research Grant TEC2006-12170/MIC. The aut thank Marta Huarte, Universidad Pública de Navarra (UPNA), Spain, and Loli Martín, SGIker at the MesostructureNanotechnology service of the Universidad del País Vasco (UPV), Spain, for their help with the measurements.

\section{REFERENCES}

[1] A. Hullmann and M. Meyer, "Publications and patents in nanotechnology," Scientometrics, vol. 58, no. 3, pp. 507-527, 2003.

[2] K. L. Choy, "Chemical vapour deposition of coatings," Progress in Materials Science, vol. 48, no. 2, pp. 57-170, 2003.

[3] M. A. Baldo, V. G. Kozlov, P. E. Burrows, et al., "Low pressure organic vapor phase deposition of small molecular weight organic light emitting device structures," Applied Physics Letters, vol. 71, no. 21, pp. 3033-3035, 1997.

[4] C. J. Lawrence, "The mechanics of spin coating of polymerfilms," Physics of Fluids, vol. 31, no. 10, pp. 2786-2795, 1988.

[5] G. G. Roberts, "An applied science perspective of LangmuirBlodgett films," Advances in Physics, vol. 34, no. 4, pp. 475-512, 1985.

[6] G. Decher, "Fuzzy nanoassemblies: toward layered polymeric multicomposites," Science, vol. 277, no. 5330, pp. 1232-1237, 1997.

[7] http://www.nanosonic.com/.

[8] K. M. Lenahan, Y. Liu, Y.-X. Wang, and R. O. Claus, "Electrostatic self-assembly processes for noncentrosymmetric thin films and devices," in Smart Structures and Materials 1999: Smart Materials Technologies, vol. 3675 of Proceedings of SPIE, pp. 104-112, Newport Beach, Calif, USA, March 1999.

[9] F. J. Arregui, Y. Liu, I. R. Matias, and R. O. Claus, "Optical fiber humidity sensor using a nano Fabry-Perot cavity formed by the ionic self-assembly method," Sensors and Actuators B, vol. 59, no. 1, pp. 54-59, 1999.

[10] F. J. Arregui, K. L. Cooper, Y. J. Liu, I. R. Matias, and R. O. Claus, "Optical fiber humidity sensor with a fast response time using the ionic self-assembly method," IEICE Transactions on Electronics, vol. E83-C, no. 3, pp. 360-364, 2000.

[11] F. J. Arregui, R. O. Claus, K. L. Cooper, C. FernándezValdivielso, and I. R. Matias, "Optical fiber gas sensor based on self-assembled gratings," Journal of Lightwave Technology, vol. 19, no. 12, pp. 1932-1937, 2001.

[12] F. J. Arregui, I. R. Matias, and R. O. Claus, "Optical fiber gas sensors based on hydrophobic alumina thin films formed by the electrostatic self-assembly monolayer process," IEEE Sensors Journal, vol. 3, no. 1, pp. 56-61, 2003.
[13] F. J. Arregui, I. Latasa, I. R. Matias, and R. O. Claus, "An optical fiber $\mathrm{pH}$ sensor based on the electrostatic self-assembly method," in Proceedings of the 2nd International Conference on Sensors, vol. 2, pp. 107-110, Toronto, Ontario, Canada, October 2003.

[14] J. Goicoechea, C. R. Zamarreño, I. R. Matias, and F. J. Arregui, "Minimizing the photobleaching of self-assembled multilayers for sensor applications," Sensors and Actuators B, vol. 126, no. 1, pp. 41-47, 2007.

[15] H. Hong, R. Sfez, E. Vaganova, S. Yitzchaik, and D. Davidov, "Electrostatically self-assembled poly(4-vinylpyridineco-vinylpyridinium-chloride)-based LED," Thin Solid Films, vol. 366, no. 1-2, pp. 260-264, 2000.

[16] M. F. Durstock, B. Taylor, R. J. Spry, et al., "Electrostatic selfassembly as a means to create organic photovoltaic devices," Synthetic Metals, vol. 116, no. 1-3, pp. 373-377, 2001.

[17] http://www.oceanoptics.com/.

[18] A. Lobnik, I. Oehme, I. Murkovic, and O. S. Wolfbeis, "pH optical sensors based on sol-gels: chemical doping versus covalent immobilization," Analytica Chimica Acta, vol. 367, no. 13, pp. 159-165, 1998.

[19] B. D. MacCraith, C. M. McDonagh, G. O’Keeffe, A. K. McEvoy, T. Butler, and F. R. Sheridan, "Sol-gel coatings for optical chemical sensors and biosensors," Sensors and Actuators B, vol. B29, no. 1-3, pp. 51-57, 1995.

[20] M. T. V. Rooney and W. R. Seitz, "An optically sensitive membrane for $\mathrm{pH}$ based on swellable polymer microspheres in a hydrogel," Analytical Communications, vol. 36, no. 7, pp. 267270, 1999.

[21] D. Westover, W. R. Seitz, and B. K. Lavine, "Synthesis and evaluation of nitrated poly(4-hydroxystyrene) microspheres for pH sensing," Microchemical Journal, vol. 74, no. 2, pp. 121129, 2003.

[22] D. Yoo, S. S. Shiratori, and M. F. Rubner, "Controlling bilayer composition and surface wettability of sequentially adsorbed multilayers of weak polyelectrolytes," Macromolecules, vol. 31, no. 13 , pp. 4309-4318, 1998.

[23] S. S. Shiratori and M. F. Rubner, "pH-dependent thickness behavior of sequentially adsorbed layers of weak polyelectrolytes," Macromolecules, vol. 33, no. 11, pp. 4213-4219, 2000.

[24] A. J. Chung and M. F. Rubner, "Methods of loading and releasing low molecular weight cationic molecules in weak polyelectrolyte multilayer films," Langmuir, vol. 18, no. 4, pp. 11761183, 2002.

[25] J. Choi and M. F. Rubner, "Influence of the degree of ionization on weak polyelectrolyte multilayer assembly," Macromolecules, vol. 38, no. 1, pp. 116-124, 2005.

[26] M. Raposo and O. N. Oliveira Jr., "Adsorption mechanisms in layer-by-layer films," Brazilian Journal of Physics, vol. 28, no. 4, pp. 392-404, 1998.

[27] M. Salomäki, I. A. Vinokurov, and J. Kankare, "Effect of temperature on the buildup of polyelectrolyte multilayers," Langmuir, vol. 21, no. 24, pp. 11232-11240, 2005.

[28] S. T. Dubas and J. B. Schlenoff, "Factors controlling the growth of polyelectrolyte multilayers," Macromolecules, vol. 32, no. 24, pp. 8153-8160, 1999.

[29] M. Yurdakoç, M. Akçay, Y. Tonbul, and K. Yurdakoç, "Acidity of silica-alumina catalysts by amine titration using Hammett indicators and FT-IR study of pyridine adsorption," Turkish Journal of Chemistry, vol. 23, no. 3, pp. 319-327, 1999.

[30] I. Del Villar, M. Achaerandio, I. R. Matias, and F. J. Arregui, "Deposition of overlays by electrostatic self-assembly in longperiod fiber gratings," Optics Letters, vol. 30, no. 7, pp. 720$722,2005$. 

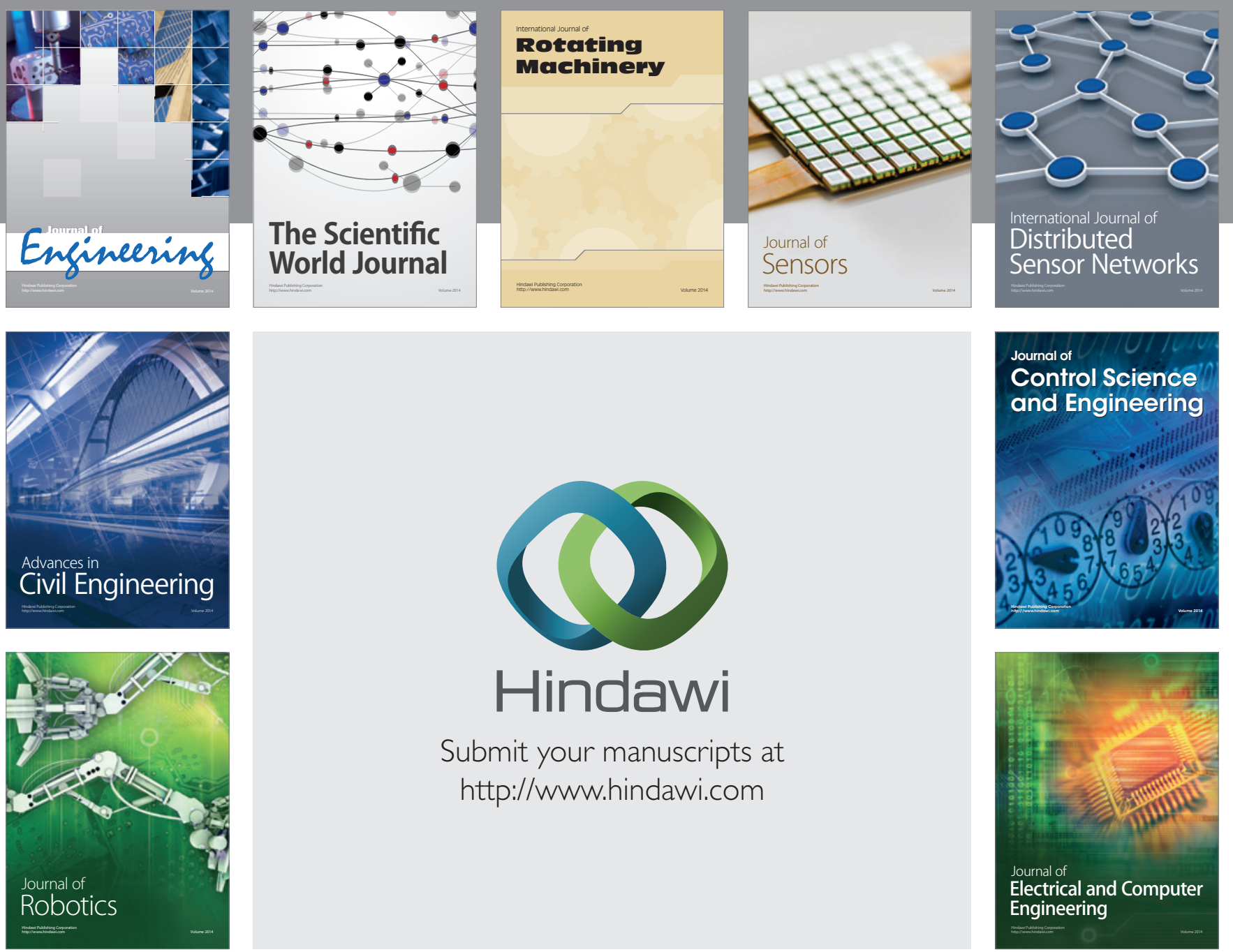

Submit your manuscripts at

http://www.hindawi.com
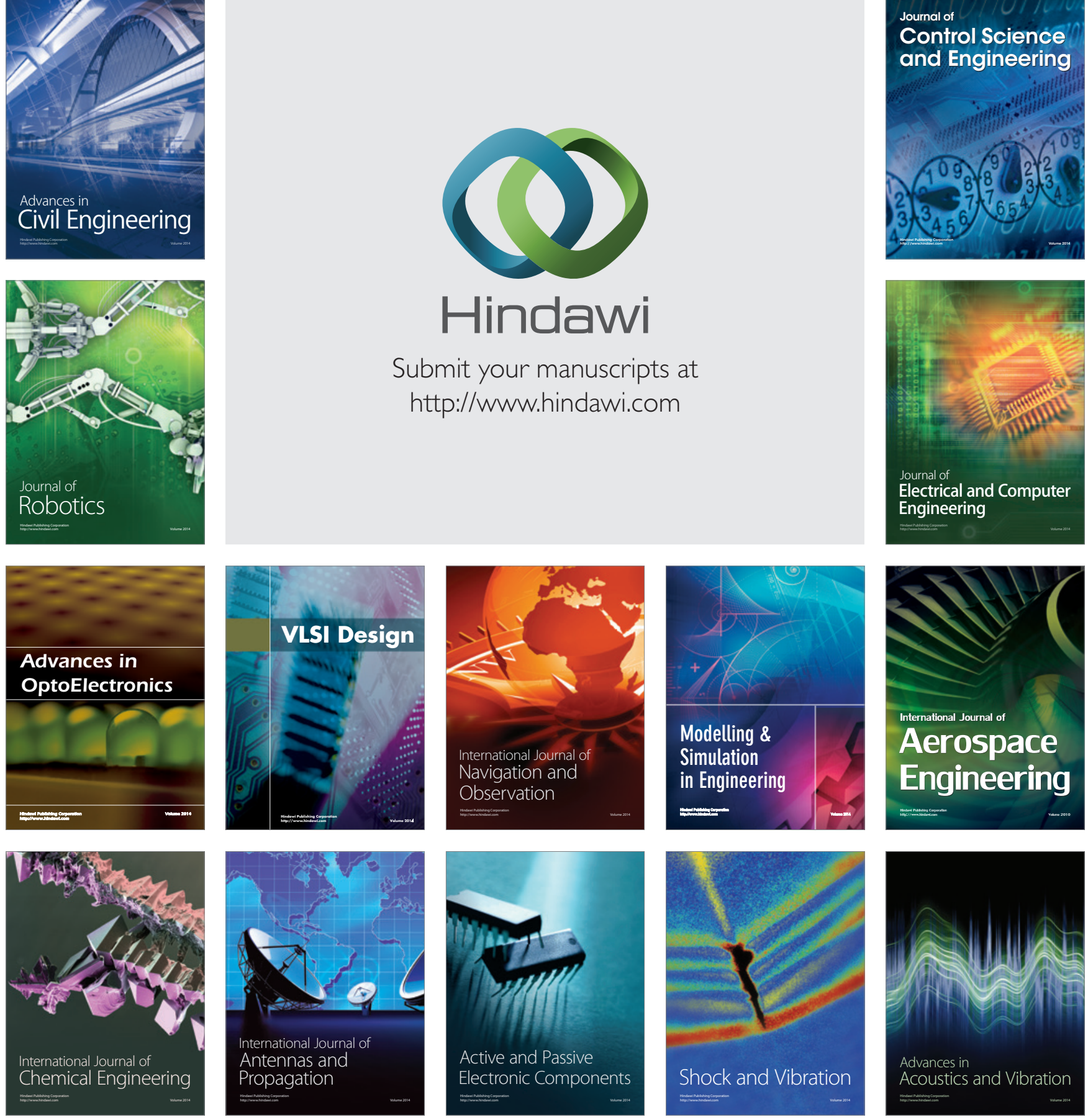